\title{
The effect of low intensity shockwave treatment (Li-SWT) on human myoblasts and mouse skeletal muscle
}

\author{
Lise K. Hansen ${ }^{1}$, Henrik D. Schrøder ${ }^{1,2}$, Lars Lund ${ }^{2,3}$, Karthikeyan Rajagopal ${ }^{4}$, Vrisha Maduri ${ }^{4}$ and Jeeva Sellathurai ${ }^{1,2^{*}}$
}

\begin{abstract}
Background: Transplanting myogenic cells and scaffolds for tissue engineering in skeletal muscle have shown inconsistent results. One of the limiting factors is neovascularization at the recipient site. Low intensity shockwave therapy (Li-SWT) has been linked to increased tissue regeneration and vascularization, both integral to survival and integration of transplanted cells. This study was conducted to demonstrate the response of myoblasts and skeletal muscle to Li-SWT.
\end{abstract}

Method: Primary isolated human myoblasts and explants were treated with low intensity shockwaves and subsequently cell viability, proliferation and differentiation were tested. Cardiotoxin induced injury was created in tibialis anterior muscles of 28 mice, and two days later, the lesions were treated with 500 impulses of Li-SWT on one of the legs. The treatment was repeated every third day of the period and ended on day 14 after cardiotoxin injection.. The animals were followed up and documented up to 21 days after cardiotoxin injury.

Results: Li-SWT had no significant effect on cell death, proliferation, differentiation and migration, the explants however showed decreased adhesion. In the animal experiments, QPCR studies revealed a significantly increased expression of apoptotic, angiogenic and myogenic genes; expression of Bax, Bcl2, Casp3, eNOS, Pax7, Myf5 and Met was increased in the early phase of regeneration in the Li-SWT treated hind limbs. Furthermore, a late accumulative angiogenic effect was demonstrated in the Li-SWT treated limbs by a significantly increased expression of Angpt 1 , eNOS, iNOS, Vegfa, and Pecam1.

Conclusion: Treatment was associated with an early upregulation in expression of selected apoptotic, proinflammatory, angiogenic and satellite cell activating genes after muscle injury. It also showed a late incremental effect on expression of pro-angiogenic genes. However, we found no changes in the number of PAX7 positive cells or blood vessel density in Li-SWT treated and control muscle. Furthermore, Li-SWT in the selected doses did not decrease survival, proliferation or differentiation of myoblasts in vitro.

Keywords: Li-SWT, Skeletal muscle regeneration, Vascularization, Angiogenesis, Myoblasts

\section{Background}

Satellite cell (Sc) derived myoblasts are widely used in regenerative medicine for engineering skeletal muscle tissue. Their successful transplantation, however, has been hampered by poor survival and poor engraftment in transplanted tissue [1-3]. Newer scaffolds have

\footnotetext{
* Correspondence: jsellathurai@health.sdu.dk; jeeva.sel@gmail.com 'Department of Clinical Pathology, SDU Muscle Research Cluster (SMRC), Odense University Hospital, Odense, Denmark

${ }^{2}$ Institute of Clinical Research, Faculty of Health Science, University of Southern Denmark, Odense, Denmark

Full list of author information is available at the end of the article
}

improved the success rates of myoblast transplantation $[4,5]$, but insufficient vascularization is still a limiting factor [6]. Common methods for improving vascularization include employment of gene-modified cells expressing angiogenic factors and scaffolds releasing angiogenic factors like VEGF and IGF1 $[7,8]$.

A promising, non-invasive, indirect technique to improve vascularisation is a low intensity shockwave treatment (Li-SWT). This is increasingly employed in regenerative medicine and wound healing [9-11] although clinical studies conducted so far have not 
advocated the use of SWT in the clinic. More research is needed to establish the clinical effect of SWT from a clinical perspective. Shockwaves are transient highpressure acoustic pulses that can be generated by different mechanical principles [12]. Shockwaves affect tissue by a direct mechanical force and by creating bubble cavitations that burst, generating a jet flow affecting nearby cells [12-14]. This results in minor intra- and extracellular damages, shear stress and conversion of mechanical force into chemical activity (known as mechanotransduction) $[14,15]$ inducing a regenerative response in the involved tissue.

In vitro studies with Li-SWT have shown to enhance certain functions involved in the behavior of specific cell types. Li-SWT increases proliferation, migration and secretion of collagenase in tenocytes treated with 1000 impulses of $0.14 \mathrm{~mJ} / \mathrm{mm}^{2}$ [16, 17]. Human osteoblasts treated with Li-SWT (500 impulses, 0.06 and $0.5 \mathrm{~mJ} / \mathrm{mm}^{2}$ ) show a dose-dependent increase in proliferation and increased expression of the genes PTHLP and PTGER3 which are involved in bone development and osteoblast differentiation [18]. Li-SWT (300 impulses, $0.1 \mathrm{~mJ} / \mathrm{mm}^{2}$ ) treated mouse endothelial progenitor cells showed increased expression of angiogenic cytokines (specifically Vegf, Nos3, Angpt1, Angpt2) and Kdr (a gene that codes for the protein vascular endothelial growth factor receptor 2) [19]. Primitive human cardiac cells showed increased KDR protein levels when treated with Li-SWT (800 impulses, $0.1 \mathrm{~mJ} / \mathrm{mm}^{2}$ ) [20].

In vivo studies with Li-SWT have shown improved tissue regeneration, greater vascularization and altered immune responses. Studies in rodents have shown increased perfusion, survival, and vessel density in skin flaps in addition to increased gene expression of $e N O S$ and $V e g f a$ [21-23], and increased perfusion of ischaemic adductor muscle [24] after Li-SWT ranging from 200 to 750 impulses, $\left(0.1 \mathrm{~mJ} / \mathrm{mm}^{2}\right)$. Altered immune response was seen in cremaster muscle tissue as decreased rolling and transmigration of leukocytes through the endothelium as well as down regulation of iNOS gene after Li-SWT (500 impulses $0.1 \mathrm{~mJ} / \mathrm{mm}^{2}$ ) $[25,26]$.

Li-SWT is a widely used treatment of tendon and bone-related conditions such as tendinopathy, plantar fasciitis and non-union of bone [27]. In addition, much ongoing research is focused on the ability of Li-SWT to ameliorate muscle pain, spasticity and peripheral arterial disease [28-30]. However, there are no published results in the indexed literature on the effect of Li-SWT on myoblast and skeletal muscle regeneration after acute injury. Thus, the question whether Li-SWT can boost regeneration and improve vascularisation of the muscle has not yet been addressed.

\section{Study objective}

The objective of this study is to address the effects of LiSWT on regenerating skeletal muscle. For this purpose we have conducted following studies:

1. An in vitro study on human myoblasts was conducted to look selectively at the effects of LiSWT on myogenic stem cells.

2. An in vivo study was conducted in mice. The animals received an acute myotoxic injury on tibialis anterior muscle, followed by treatment with Li-SWT to study the effect of Li-SWT on muscle regeneration.

\section{Methods}

\section{Shockwave application}

A handheld Duolith SD1 equipment (Storz, Tägerwilen, Switzerland) was set at the intensity $0.10 \mathrm{~mJ} / \mathrm{mm}^{2}, 5 \mathrm{~Hz}$ (5 impulses pr. second), with $300,500,1000$, or $1500 \mathrm{im}$ pulses given continuously in one treatment lasting 60s, 100 s, 200 s, or 300 s respectively. The treated area was covered with EKO GEL (ultrasound transmission gel, Ekkomarine Medico A/S, Denmark) and the focused shockwaves were delivered with a focal area (penetration depth) at 0-30 $\mathrm{mm}$.

For the in-vitro experiments, a cryotube $(1.8 \mathrm{ml})$ filled with Ultroser G (UG) medium containing $2.5 \times 10^{5}$ cells and a cryotube filled with growth medium (GM which consisted of DMEM with 10\% FBS and 1\% PSA (Penicillin-Streptomycin-amphotericin B, Life Technologies)) containing pieces of muscle explants of approx. $0.5 \mathrm{~cm}^{3}$. These were covered with EKO GEL and treated once with $300,500,1000$ or 1500 impulses of Li-SWT immediately before culturing.

The control cells were transferred to cryotubes at the same time as the samples treated with Li-SWT before culturing, but did not receive shockwave treatment.

\section{Myoblast isolation and culturing}

Human myoblasts were isolated from biopsies taken from the vastus lateralis muscle of young men (1820 years). These samples were obtained from a previous study [31]. Biopsies free of connective tissue were minced and digested with $0.3 \%$ collagenase type II (Medinova Scientific) for $40 \mathrm{~min}$. in $37{ }^{\circ} \mathrm{C}$ water bath. The suspension was titrated with a $1 \mathrm{ml}$ pipette, cold HBSS (Hanks Balanced Salt Solution) with 10\% FBS was added and the suspension was pelleted and re-suspended in $37{ }^{\circ} \mathrm{C}$ HBSS with $10 \%$ fetal bovine serum (FBS) and filtrated first through a $100 \mu \mathrm{m}$ then through a $40 \mu \mathrm{m}$ Falcon Cell Strainer. The isolated satellite cells were cultured in GM and plated on extracellular matrix (ECM, Sigma-Aldrich, MO, USA) coated dishes (Nunclon, Nunc). During every passage, the number of 
fibroblasts was reduced by pre-plating the cells for $20 \mathrm{~min}$. at $37{ }^{\circ} \mathrm{C}$ on untreated NUNC dishes. The nonadherent cells were harvested, expanded and aliquots were frozen and kept in liquid nitrogen.

For the in vitro experiments the cells were thawed and seeded on ECM (Extracellular Matrix, Sigma-Aldrich, MO, USA) coated flasks and coverslips (Fischer Scientific, MA, USA) and cultured in growth medium (DMEM w. hepes and DMEM w. glutamat (Life Technologies, CA, USA)) with $2 \%$ Ultroser G (Pall), 2\% FBS (Fetal bovine serum, Life Technologies) and 1\% PSA (Penicillin-Streptomycin-amphotericin B, Life Technologies).

\section{Explant culture conditions}

Human explants were obtained from the m.quardriceps femoris and $m$. soleus (females aged 78 and 31 ), and cultured in a growth medium with 10\% FBS and 1\% PSA. The media was changed 9 days after Li-SWT and afterwards twice a week and the explants were harvested after 17 days.

\section{Myoblast viability, proliferation and differentiation after Li-SWT}

Myoblast survival was measured by tryphan blue staining (Sigma-Aldrich) immediately after shockwave treatment. In order to examine the proliferation rate after LiSWT, myoblasts were cultured in $10 \mathrm{mM}$ BrdU in UG media (cumulative BrdU incorporation), and coverslips were harvested at days 1, 2, 3 and 4 after treatment. The differentiation ability of myoblasts after Li-SWT was tested by culturing them in a differentiation medium (DMEM with 2\% FBS, 1\% PSA and 25 pM insulin (Actrapid from Novo Nordisk, DK)) and cells were harvested on days 2, 4, and 6 after Li-SWT.

\section{Animal experiment}

Twenty-eight female mice aged 10 weeks (C57BL/ $6 \mathrm{NTac}$ ) received a single lesion in both of their tibialis anterior muscle (TA) through an injection of $50 \mu \mathrm{l}$ cardiotoxin, CTX $(10 \mu \mathrm{M})$. Two days later the mice were treated with Li-SWT for $100 \mathrm{~s}$ (500 impulses of $0.1 \mathrm{~mJ} /$ $\mathrm{mm}^{2}$ (5 Hz), 1.635 joule) on the left leg, while the right leg functioned as control. Prior to the shockwave application, the skin of the leg was depilated to eliminate shockwave interferences. The treatment was repeated every third day of the period, ending on day 14 after CTX injection. Four mice were euthanized at each time point on days 2 ( $5 \mathrm{~h}$ after first Li-SWT treatment), 3, 5, $7,10,14$ and 21 after cardiotoxin injection.

The TA muscles were removed from both legs and cut into halves. One half was stored in RNAlater (Ambion, Life technologies) in $-20 \mathrm{C}^{\circ}$ for $\mathrm{qPCR}$ analysis. The other half was fixed in formalin for $24 \mathrm{~h}$ and embedded in paraffin for histological studies.

\section{RT-qPCR}

\section{Cell cultures}

Gene expression studies were performed with 3 biological replicates.

In order to examine gene expression under proliferation, cell cultures were harvested $5 \mathrm{~h}, 12 \mathrm{~h}, 24 \mathrm{~h}, 48 \mathrm{~h}$, $72 \mathrm{~h}$ and $96 \mathrm{~h}$ after Li-SWT and lysed with $0.5-1 \mathrm{ml}$ trizol (Life Technologies, CA, USA).

\section{Animal experiments}

Of the four mice euthanized, three were used for RTqPCR. The muscles were lysed in $1 \mathrm{ml}$ trizol using MagNA lyser beads and processed on Magna Lyser Instrument (Roche Applied science, DK) for $2 \times 20 \mathrm{~s}$ at $6500 \mathrm{rpm}$, following this RNA was extracted.

cDNA was synthesized from 500 ng RNA using High Capacity cDNA Reverse Transcription kit (Life Technologies) and qPCR performed on Quantstudie $12 \mathrm{~K}$ flex (Life Technologies) using custom designed 384 well TaqMan Array Micro Fluidic Cards. The data were analysed in qBasePlus (Biogazelle), and quality control was set by excluding replicates with $>0.5$ deviation from quantification cycle $(\mathrm{Cq})$. Reference genes were selected using Genorm ( $\mathrm{M}$-value <1.5). The results for the individual genes are shown as mean fold change to the lowest normalised $\mathrm{Cq}$ value, which was set to 1 . The following genes were examined in myoblast cultures: TGFb1, SMAD3, SMAD7, MKI67, CYCLIND1, P21, TP53, CASP3, PAX3, PAX7, MYF5, MYOD1, MYOG, MEF2A, $M E T, H G F$ and $K D R$. GAPDH and TBP were used as reference genes.

In the animal experiment the genes examined were: Bax, Bcl2, Casp3, Il1a, Il1b, Il6, Ccl2, Ccr2, Tnf, Pax7, Myf5, Myod1, Myog, Myf6, Mstn, Met, Hgf, Mef2c, Tgfb1, Angpt1, Angpt2, iNOS, eNOS, Vegfa, Kdr, Pecam1, Cd34, Fgf2 and Fgfr1. Rn18s, Tbp and Tfrc were used as reference genes. All qPCR data (fold changes) for the in vitro and in vivo experiments can be seen in Additional file 1.

\section{Immunohistochemistry \\ Cell cultures}

For detection of BrdU, samples were fixed in 96\% alcohol for 30 min before incubation in $2 \mathrm{~N} \mathrm{HCL}$ with $0.5 \%$ Triton X-100 for $30 \mathrm{~min}$. Afterwards samples were neutralised for $3 \times 5 \mathrm{~min}$ in $\mathrm{NaBH}_{4}$ solution $(1 \mathrm{mg} / \mathrm{ml})$, followed by incubation of mouse-anti-BrdU (1:20, Bu20a clone, Dako, DK).

For detection of NCAM, explant samples were fixed in 4\% formalin for $5 \mathrm{~min}$ followed by incubation in Triton X-100 for $5 \mathrm{~min}$, prior to incubation with mouse-antiNCAM (1:100 CD56 Leu-19), Becton Dickinson, DK).

For detection of myogenin, samples were fixed $15 \mathrm{~min}$ in $4 \%$ formalin followed by incubation in $96 \%$ ethanol for $10 \mathrm{~min}$. After rinsing in water the samples were 
incubated in Tris-EGTA buffer at $95{ }^{\circ} \mathrm{C}$ for $15 \mathrm{~min}$, followed by incubation with mouse-anti-myogenin (1:800, F5D, Dako). Powervision (Leica Biosystems) was used for detecting BrdU, NCAM and myogenin.

For detection of F-actin, samples were washed twice in preheated $\left(37^{\circ}\right)$ TBS (Tris-buffered saline) and fixed in $4 \%$ formalin for $10 \mathrm{~min}$ and incubated in $0.1 \%$ TritonX100 for $5 \mathrm{~min}$, before incubation in phalloidin (1:40, Alexa Flour 546 Phalloidin, Lifetechnologies). After washing with PBS, samples were mounted with Vectashield (Vector Lab, UK).

\section{In vivo experiment}

Staining of muscle paraffin sections was carried out on Dako Autostainer Plus (Dako) using Dako EnVision + kit and the following antibodies: rabbit anti-rat IgG (Dako) followed by rat-anti-Cd45 (1:100, 30-F11, Pharmingen, BD, DK), rabbit-anti-vWF (1:2000, Dakopatts A/S, DK), mouse-anti-myogenin (1:200, clone F5D, Dako) and mouse-anti-Pax7 (1:20, Hybridoma Bank, IA, USA). The sections were blocked for endogen biotin by Avidin/Biotin Blocking kit (Vector Lab, UK) prior to incubation with myogenin and pax7.

\section{Morphometrics and statistical analyses}

CAST software (Visiopharm A/S, Hørsholm, DK) was used for all morphometric analysis. Systematic, random counts were performed on blinded samples including the entire cell containing area. In assessment of BrdU incorporation and cell adhesion $10 \%$ of the coverslip area was counted, while $25 \%$ was counted when quantifying myofibers ( $\geq 3$ myogenin + nuclei) and $2 \%$ when determining the myogenin fraction in cell cultures.

Quantification of the immunostained paraffin sections from the animal experiment was performed by counting MYOG+, PAX7+ and CD45+ cells and vWF+ vessels in $100 \%$ of the selected areas (regenerating and nonregenerating part of the TA muscle). As the size of the muscle injury induced by cardiotoxin varied between animals, the selected regenerating area varied, too, why the data is presented as positive events per $\mathrm{cm}^{2}$.

Statistics were performed in Graphpad Prism, version 5.0 (Graph Pad Software Inc., LA, USA). All data are shown in mean + SD (standard deviation) and results are tested by paired t-test in order to detect difference between Li-SWT treatment and control. P-values below 0.05 were considered significant.

\section{Results}

In vitro cell survival, proliferation and apoptosis of myoblasts were not affected by Li-SWT

Cells were treated with 300, 500, 1000 and 1500 impulses of Li-SWT and cell death subsequently quantified by tryphan blue uptake. Cell death was between 2.8-
$5.6 \%$ in the Li-SWT treated compared to $1.7-3.0 \%$ in controls, and thus we found no significant difference in cell death (Fig. 1a).

The effect of Li-SWT on myoblast proliferation was assessed by counting the number of cells on day $1,2,3$ and 4 after treatment. The results showed some variation between the three cell cultures, but overall no significant difference between shockwave treated and controls was observed (Fig. 1b), nor did the BrdU incorporation studies demonstrate increased proliferation in treated cells compared to controls (Fig. 1c).

The expression of TGFB1 during proliferation was significantly decreased at $12 \mathrm{~h}$ in the Li-SWT group, while $S M A D 7$ in this group at $12 \mathrm{~h}$ was significantly increased, yet there was no difference between the groups in SMAD3 and CASP3 expression (Fig. 1d). The cell cycle related genes KI67, CYCLIND1 and P53 showed no difference in expression (Fig. 1d), except P21 was significantly different at $12 \mathrm{~h}, 24 \mathrm{~h}$ and $72 \mathrm{~h}$. Thus, the data supports that Li-SWT exerted neither negative nor positive effects on cell survival and proliferation of myoblasts.

No difference was observed in the expression of the myogenic factors PAX3, PAX7, MYF5, MYOD1, MYOG, $M E T$, and $H G F$ genes during proliferation. However, $M E F 2 A$, a co-actor in myoblast differentiation [32], was significantly increased at $12 \mathrm{~h}$ in the Li-SWT group compared to control (Fig. 2).

\section{Li-SWT did not influence the migration ability in vitro}

A scratch test performed $48 \mathrm{~h}$ after Li-SWT treatment with a duration of $18 \mathrm{~h}$ revealed no change in the ability of human Li-SWT myoblasts to migrate compared to controls. (See Additional file 2 for images and detailed method).

\section{Li-SWT did not alter myogenesis in vitro}

In order to determine if shockwaves affect the differentiation, myofiber (>2 nuclei) formation was quantified after culturing in differentiation medium for 2, 4 and 6 days. As an additional parameter the fraction of MYOG positive nuclei was estimated. Though no difference was seen in presence of myofibers (Fig. 3a), the fraction of MYOG+ cells was significantly lower on day 2 in the Li-SWT group (Fig. 3b). Although the number of MYOG+ cell thus seemed lower during induction of differentiation, the capacity for myofiber formation and the overall ability to differentiate was not impaired by Li-SWT.

\section{Decreased adhesion of explants after Li-SWT}

We found that $46 \%$ of the Li-SWT treated explants compared to $92 \%$ in controls $(n=48)$ adhered to the 


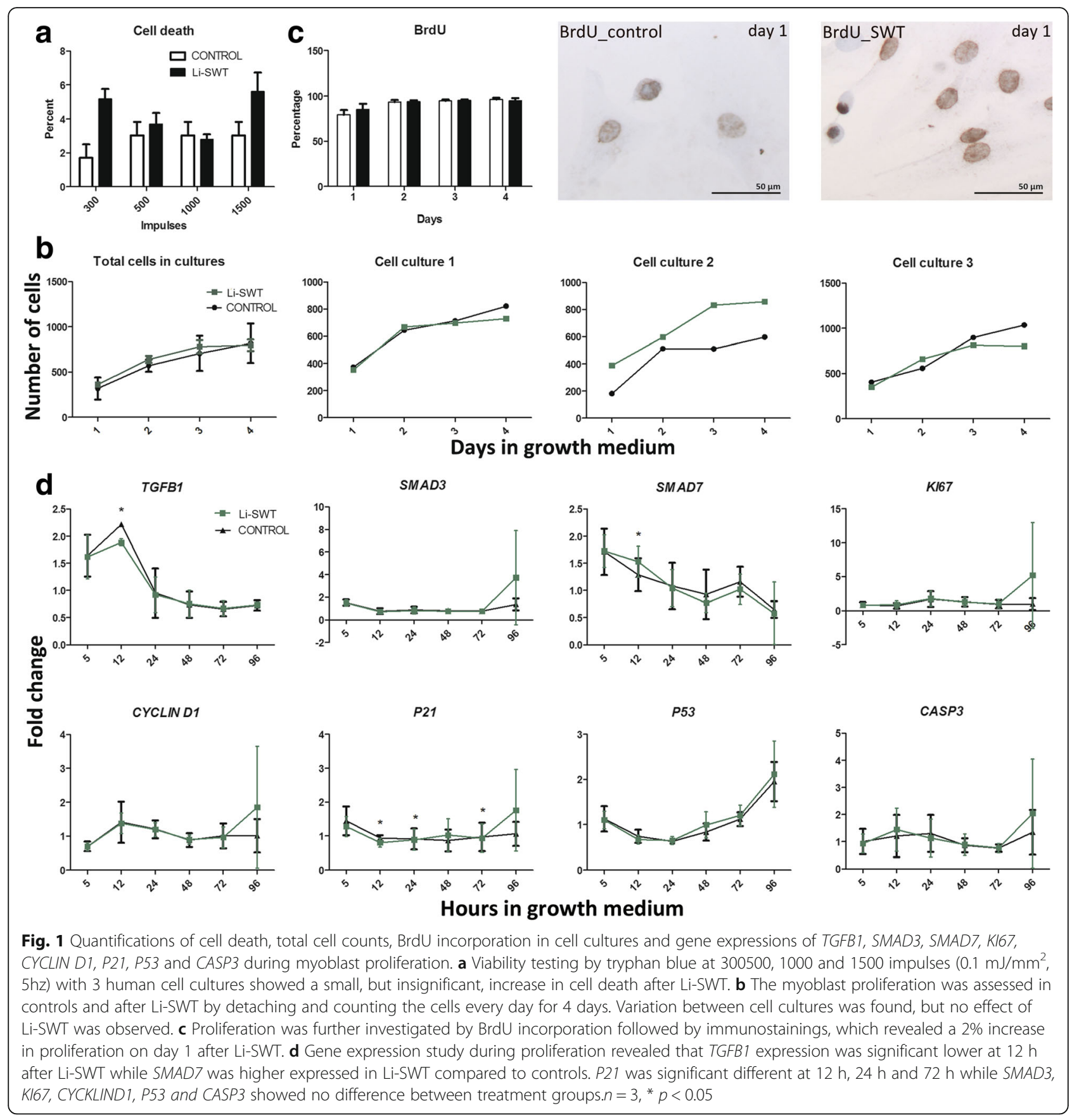

coverslips after 7 days (Fig. 4a). However, no difference was seen in the extent of outgrown cells or NCAM protein expression in the Li-SWT group compared to controls (Fig. 4b).

The adhesion kinetics of primary isolated myoblasts (isolated by enzyme dissociation) were then tested by treating the cells with Li-SWT followed by cell harvest every $30 \mathrm{~min}$ for the first $2 \mathrm{~h}$ after seeding. No reduced adherence following Li-SWT was seen in treated cells compared to controls (Fig. 4c.). However, the expression of F-actin, visualized by phalloidin staining, was reduced in Li-SWT treated cells, indicating a delayed formation of F-actin (Fig. 4d).

\section{In vivo}

The effects of Li-SWT on skeletal muscle regeneration were studied in mouse muscle using a cardiotoxininduced lesion that provides a complete regenerating microenvironment. The cardiotoxin lesion did not cover the entire TA muscle, hence the histological sections contained partly normal and partly regenerating area (Additional file 3). 


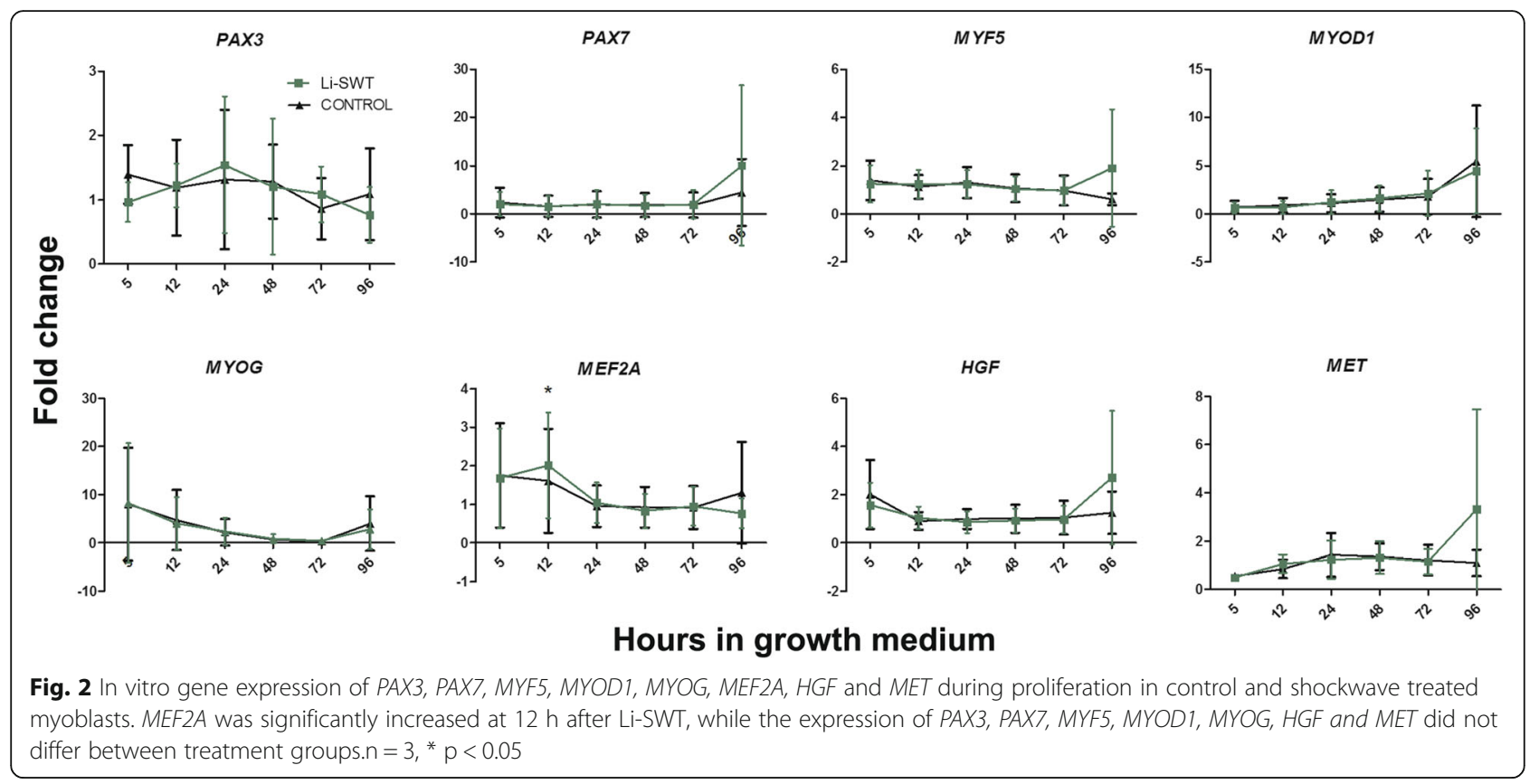

Expression of apoptosis related genes increased during early regeneration in shockwave treated animals Expression of the apoptosis related genes $\mathrm{Bax}, \mathrm{Bcl} 2$ and Casp3 increased significantly on day 2 in shockwave treated legs compared to controls, indicating a stronger onset of apoptosis. The $\mathrm{Bax} / \mathrm{Bcl} 2$ ratio, which determines the apoptotic potential [33], peaked at day 3 and decreased until day 10 for both groups, reflecting the muscle degeneration observed as part of the regeneration process [34, 35] (Fig. 5a).
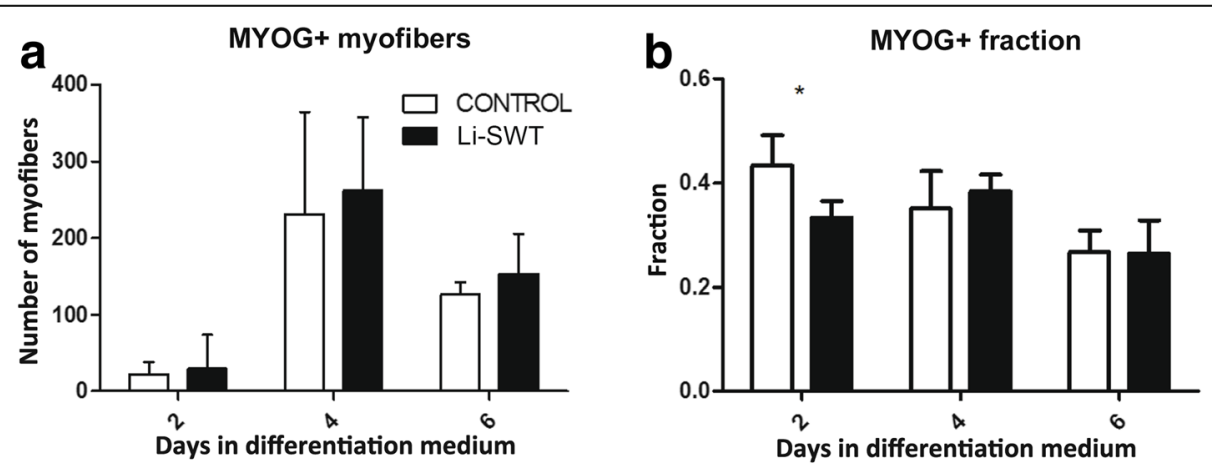

C
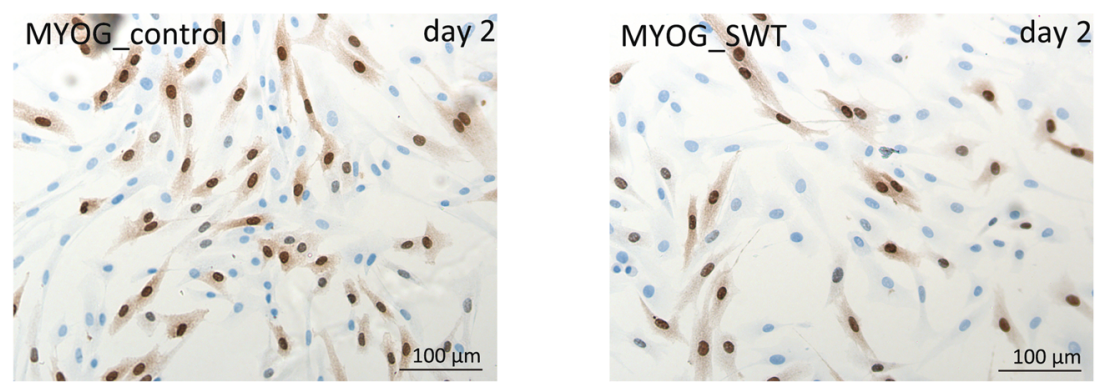

Fig. 3 The number of MYOG positive myofibers and the fraction of MYOG positive cells during myoblast differentiation after Li-SWT. Myoblasts were treated with shockwaves and cultured in differentiation medium and harvested 2, 4 and 6 days after Li-SWT. a We found no difference in the number of myofibers (>2 nuclei) in control and Li-SWT myoblasts, thus cells had maintained their capacity to differentiate after Li-SWT. $\mathbf{b}$ The fraction of MYOG positive nuclei was significantly lower in Li-SWT cultures on day 2 during differentiation. However on day 4 and 6 , the MYOG fractions were not changed by Li-SWT. c Immunohistochemical stainings for MYOG on day 2 after Li-SWT. $n=3,{ }^{*} p<0.05$ 

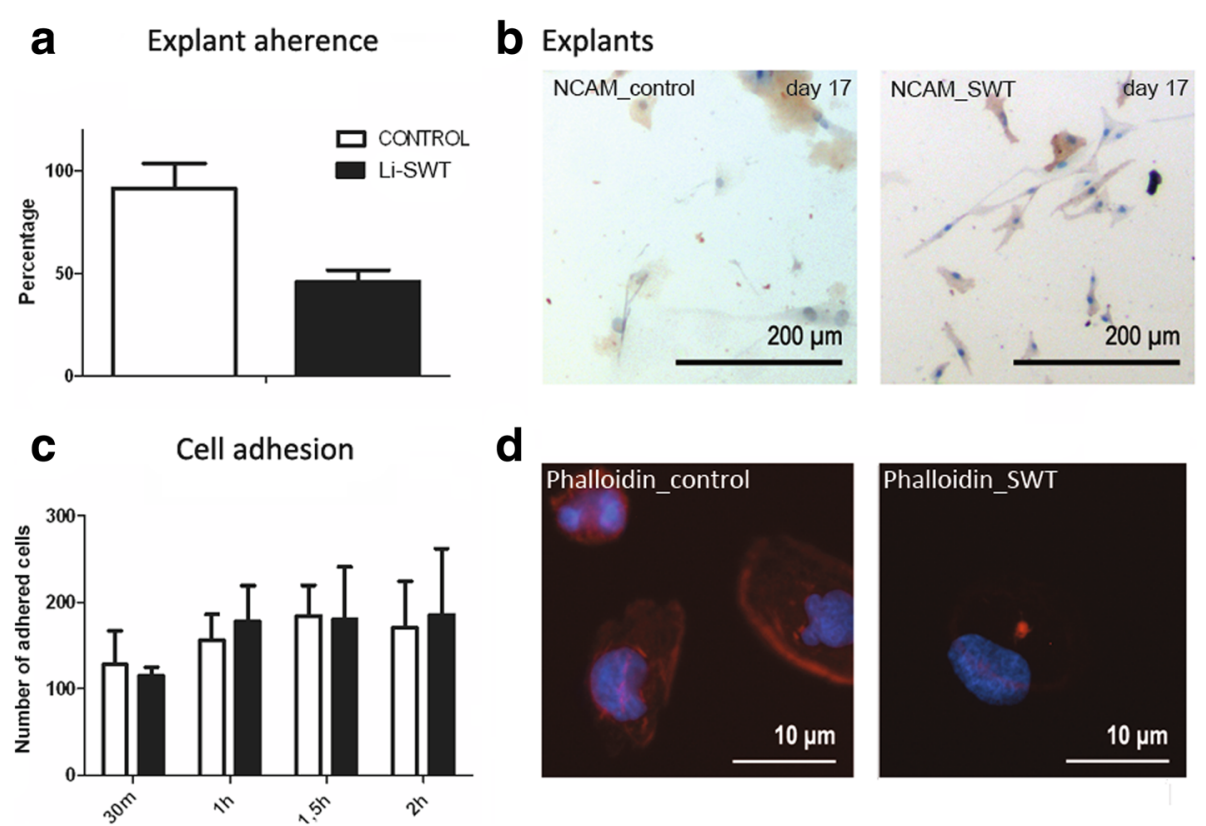

d

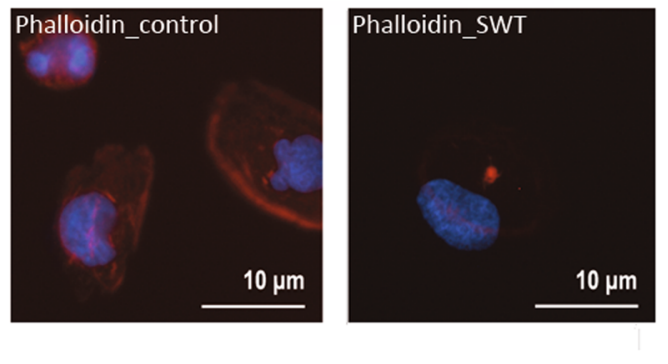

Fig. 4 Quantifications of adherence of explants, outgrowth visualisations, cell adhesion and phalloidin staining of cells after shockwave treatment. a In explant cultures treated with 300 impulses of $0.1 \mathrm{~mJ} / \mathrm{mm}^{2} 46 \%$ of the treated explants adhered compared to $92 \%$ in controls ( $n=48$ ), however (b) the number of cells grown out from the explants and their expression of NCAM did not differ. $\mathbf{c}$ The experiment was then performed with human myoblasts isolated by enzyme dissociation. The cells were cultured on coverslips and harvested every 30 min the first 2 h; no difference in adherence was observed. (d) However, when the harvested coverslips were stained for F-actin with phalloidin, the shockwave treated cells displayed a much lower expression of F-actin, indicating a delayed actin assembly. The shown images are from cells harvested 90 min after culture. $n=3,{ }^{*} p<0.05$

\section{Small effect of Li-SWT on the early inflammatory response} The inflammatory response induced by the cardiotoxin lesion caused a significant increased Illa expression on day 2, and Tnfa on day 3 in the Li-SWT legs, but no difference in the expression of $I l 1 b, I l 6, C c l 2$ and $C c r 2$ was found between the groups. All genes were initially expressed at high levels and decreased towards day 10 with no further changes (Fig. 5a).

CD45 staining for inflammatory cells on the histological sections demonstrated a lesion-induced inflammatory cells on day 2 and 3, but no difference was observed between the groups except on day 21 where the infiltration was significantly higher in the Li-SWT legs (Fig. 5b, c).

Thus, a small increase in the early inflammatory signal may have occurred; however, the overall inflammatory response was not altered by the Li-SWT.

\section{Li-SWT caused upregulation of myogenic factors in vivo} Pax7, Myf5 and Met gene expressions were significantly increased on day 2 in the Li-SWT group. No difference was observed in the expression of Myod1, Myog, Myf6, Mstn, Hgf, Mef2c and Tgfb1 (Fig. 6a).

Immunohistochemical stainings for the satellite cell marker PAX7 was made (Fig. 6b) and the stainings were quantified on day 2 and 3 (Fig. 6c). As expected a higher density of PAX7 positive cells was observed in the regenerating areas, however we found no difference in the number of PAX7 + cells between the treated and control legs. Likewise, a large number of MYOG+ cells were observed in the regenerating areas on day 2 and 3, however no difference between Li-SWT and controls was found (Fig. 6d).

Thus gene expression studies indicate a Li-SWT induced early SC activation though the number of cells expressing the myogenic markers PAX7 and MYOG was unaltered.

\section{Li-SWT increased the angiogenic gene expression in vivo}

The gene expression of Angpt1, Angpt2, eNos and iNos were high on day 2 in the Li-SWT group. eNos was significantly increased in the Li-SWT group on day 2 and iNos and Angpt2 showed the same tendency (Fig. 7a). This could indicate that Li-SWT supports early angiogenesis during muscle repair. On day 21 Angpt1, eNos, iNos, Vegfa, and Pecam1 were significantly higher expressed in Li-SWT treated legs, with Angpt2 and Vegfr2 having the same tendency (Fig. 7a), indicating a late effect, too, of Li-SWT, corresponding to previous studies [21, 36].

CD34 gene expression increased slightly in both groups from day 10, but there was no difference between 


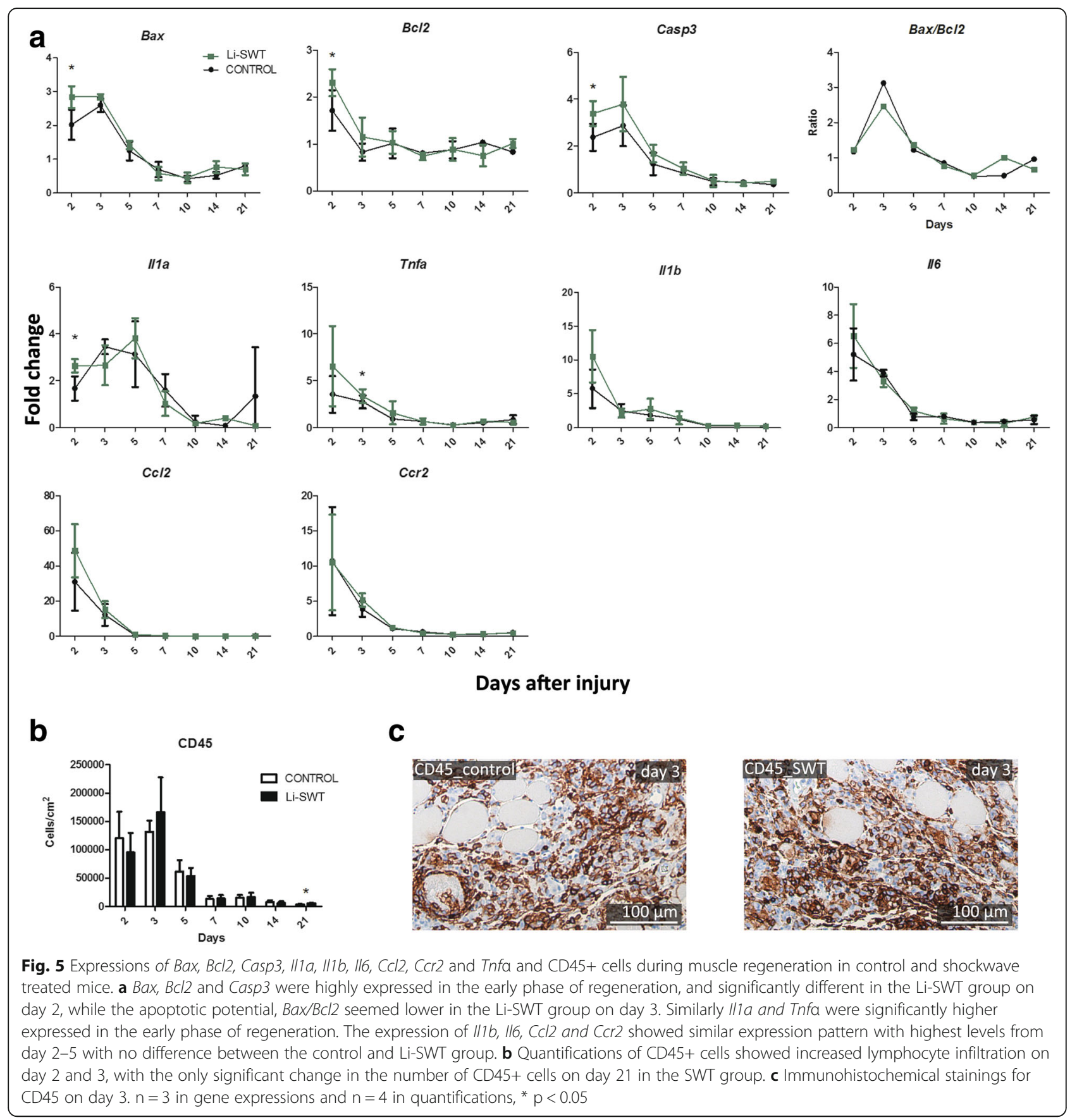

treated and control legs. Furthermore there was no difference in the Fgf2 expression, a well-known co-player in angiogenesis [37], while the expression of Fgfr1 was significantly increased on day 2 in Li-SWT treated compared to control (Fig. 7a).

Quantitation of vessel density by immunohistochemial staining with vWF demonstrated increased vessel density in the regeneration area compared to the unaffected area of the muscle, but there was no difference between treatment groups (Fig. 7b,c).
Though no difference in vessel density was found, the gene expressions indicate an enhanced angiogenic signal caused by Li-SWT.

\section{Discussion}

Our study is the first to present the effect of LiSWT on human myoblast cultures and mouse skeletal muscle undergoing regeneration after acute injury. The beneficial effect of Li-SWT treatment on microcirculation has been documented in various 


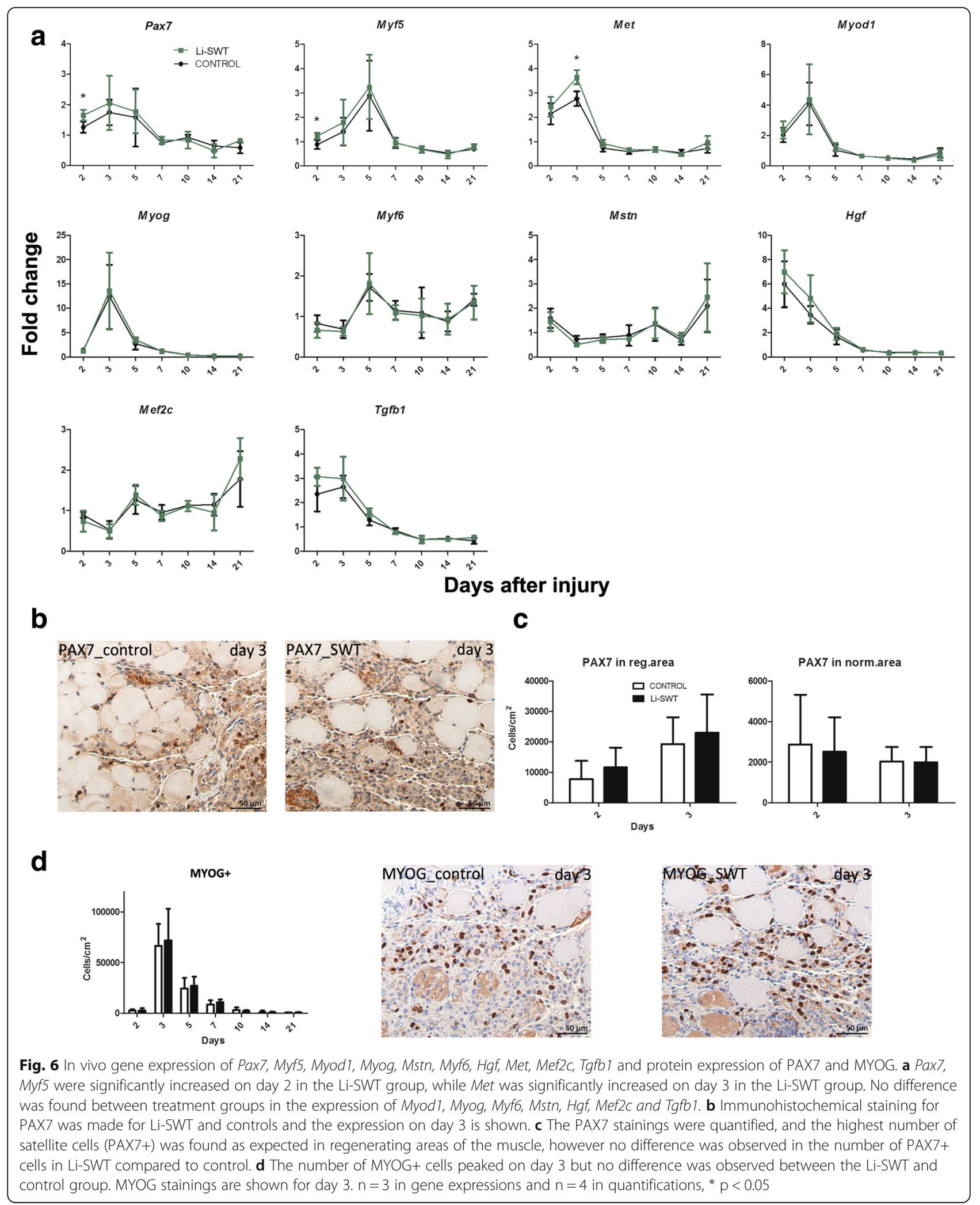

tissues [21, 25, 38, 39] but not much is known about how Li-SWT affects the skeletal muscle specific stem cells.
Li-SWT exerted no harmful effect on human myoblasts

The viability of myoblasts was tested, and within the range of 300 to 1500 impulses the energy application 


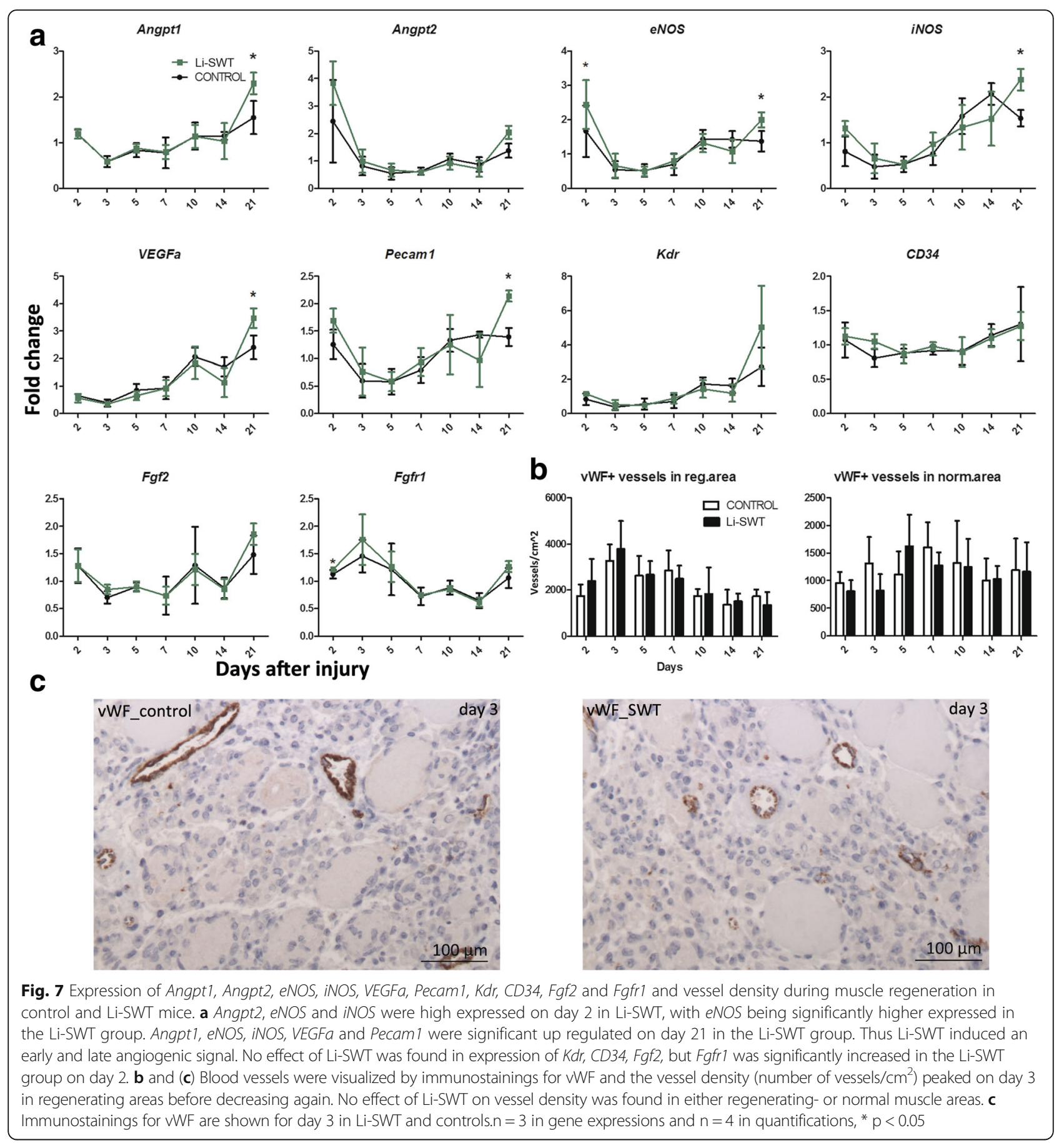

was well tolerated. For further in vitro studies a dose of 300 impulses of $0.1 \mathrm{~mJ} / \mathrm{mm}^{2}$ was used. Similar in vitro studies have used impulses ranging from 250 to 1000 depending on cell type $[17,19,40]$. When myoblast cultures were treated with Li-SWT we found a very low level of cell death, comparable to studies conducted on osteoblasts and fibroblasts [40]. We also found that the proliferation potential of myoblasts and their ability to migrate and differentiate was unaffected by the Li-SWT with the applied dose. Thus Li-SWT was not harmful to myoblasts.

\section{Li-SWT altered expression of TGF $\beta 1$, SMAD7 and MEF2A during in vitro proliferation}

Although Li-SWT had no effect on the number of proliferating cells, we found that the expression of TGF 1 was down regulated while $S M A D 7, P 21$ and $M E F 2 A$ were significantly up regulated in the Li-SWT treated cell 
cultures during proliferation. Thus, the TGF $\beta 1$ signaling pathway seemed to be sensitive for shockwave treatment, however no conclusion on the overall influence of the pathway can be drawn from the present gene expression data. $M E F 2 A$ is crucial for myoblast differentiation [32], and interestingly, it was significantly up regulated already $12 \mathrm{~h}$ after treatment. In $\mathrm{C} 2 \mathrm{C} 12 \mathrm{MEF} 2 \mathrm{~A}$ is increased after mechanical stress in $\mathrm{C} 2 \mathrm{C} 12$ myoblasts [41], thus the Li-SWT induced shear stress may have led to the up regulation of MEF2A in the human myoblasts.

\section{Myoblast stress tolerance}

It is likely that various cell types can withstand different amount of stress. Tenocytes are often treated with 1000 impulses [16, 17], while cells of endothelial origin, which are sensitive to flow changes (shear stress) [42], tend to be treated with 300 impulses [19, 43]. Myoblasts may have a stress tolerance similar to tenocytes given their similar niche and ability to sustain continues stretch, which could explain the lack of effects of Li-SWT on proliferation and differentiation observed in this study.

\section{Decreased adhesion in explants and cell cultures after Li-SWT}

Stress mechanotransduction can affect the cytoskeleton and focal adhesion proteins, leading to loss of adhesion [44]. Likewise, shockwave treatment has been reported to result in cell detachment in monolayers of cardiac cell cultures [20] and renal carcinoma cell line [45],. However, increased adhesion has been reported in suspended osteoblasts treated with Li-SWT [46]. Thus, the effect of Li-SWT seems to depend on the method of application and cell type.

The decreased attachment of Li-SW treated explants observed in our study could be due to an initial effect of Li-SWT. Detached renal carcinoma cells showed actin depolymerisation and altered actin filament organisation [45]. Likewise, we observed a decreased assembly of Factin after Li-SWT. Although we did not observe altered attachment properties, this indicates an effect of Li-SWT on myoblast cytoskeleton.

\section{Li-SWT affected the muscle regeneration in mice}

In our study Li-SWT significantly increased apoptotic, pro-inflammatory and myogenic factors in the early phase of regeneration, probably a result of shockwaveinduced stress in an already necrotic/apoptotic environment [33].

Although the $B a x / B c l-2$ ratio indicated that apoptotic activity was reduced on day 3 in the treated legs, the significant expression of Bax and Casp3 on the previous day implied a Li-SWT-induced pro-apoptotic gene expression. Other studies have reported decreased necrosis and decreased amount of cells undergoing apoptosis in ischaemic skin flaps after Li-SWT [22, 47], however, an ischaemic skin flap is different from regenerating muscle in an otherwise healthy mouse, and the response upon injury depends on the type and the extend of the injury, for instance degeneration and regeneration progress faster after myotoxic injury than after ischaemic lesions [35, 48].

In the inflammatory markers Illa and Tnfa was significantly increased in early regeneration and $I l 1 b$ and $I l 6$ showed the same tendency. However, quantifications of leucocytes did not reveal increased infiltration nor was there any increase in the $\mathrm{Ccl} 2$ expression, indicating similar monocyte/macrophage infiltration [49]. In contrast a recent study reported significantly decreased expression of pro-inflammatory genes and leukocyte infiltration in burn injuries after Li-SWT [50] and another study reported decreased leukocyte rolling and transmigration of endothelium after Li-SWT [25].

Though Li-SWT did not augment the number of PAX7 + satellite cells, an increased expression of Pax7, Myf5 and Met on day 2, indicate increased SC induction. Thus LiSWT may have induced the regenerative response in the acute muscle injury model, in accordance with reported observations on the effect of pro-inflammatory cytokines, HGF release and shear stress [34, 51-53]. The effect might prove even more beneficial in a chronic injury model, where the background is suboptimal repair.

\section{Increased angiogenic response in mice}

We found in the regenerating muscle an increased expression of $e N O S$ on day 2 after Li-SWT and the same tendency was observed in $i N O S$ and Angpt2, indicating an early angiogenic reponse. Furthermore, Angpt1, eNOS, iNOS, Vegfa, and Pecam1 gene expressions increased from day 14 to 21 in the Li-SWT group,

The increased eNOS and iNOS expression was most likely caused by shockwave-induced increase in intracellular calcium, which activates eNOS and $i N O S$ [54]. Further, iNOS is induced by pro-inflammatory cytokines like TNF-a and IL1b [55]. In addition, shear stress is known to contribute to increased eNOS expression [56] and increased release of angpt 2 from endothelial cells [57], which could potentially lead to increased angiogenesis.

Despite Li-SWT induction of pro-angiogenic genes the vessel density was unaffected, which is in contrast to recent studies reporting increased vessel density after treatment of ischaemic muscle or skin $[23,24,26]$. Thus, the injury model, might be crucial for SW-induced acceleration of regeneration. The mice used in the present study were healthy and thus fully capable of regenerating a cardiotoxin lesion without Li-SWT. To be demonstrable a Li-SWT induced angiogenic response may need to be studied in a disease model where the regeneration capacity as a starting point is insufficient. 
In a clinical perspective the increased pro-angiogenic gene expression and the early stimulation of satellite cells induced by Li-SWT might be beneficial in regenerating skeletal muscle injuries. Especially the field of skeletal muscle tissue engineering with the use of scaffolds where activation of myoblasts and proper vascularization are limiting factors for survival and integration of transplanted tissue, treatment with Li-SWT might enhance the engraftment and regeneration process. Likewise support of regeneration in muscle lesions in areas with insufficient blood supply might be a target for Li-SWT.

Shockwave treatment is commonly applied to tissues covered by or adjacent to muscle and thus skeletal muscle is included in the treatment area. It is therefore an important information that muscle apparently is not injured by shockwave treatment.

Many articles have reported the effect of Li-SWT, but this study describes a large number of genes affected by Li-SWT, which to our knowledge has never been described before.

\section{Conclusions}

Our studies have shown, that Li-SWT is well tolerated and that when applied to muscle stem cells in vitro and muscle in vivo with a total energy of $0.981 \mathrm{~J}$ and $1.635 \mathrm{~J}$ respectively cellular changes were induced that could promote muscle regeneration.

\section{Additional files}

Additional file 1: Numerical data (fold changes) from the in vitro and in vivo gene expression studies. The table shows the numerical data (fold changes) from the in vitro gene expression studies (Fig. 1 and Fig. 2) and the numerical data from the in vivo gene expression studies (Fig. 5, Fig. 6 and Fig. 7). (XLSX $58 \mathrm{~kb})$

Additional file 2: Migrating ability after Li-SWT. A scratch test performed $48 \mathrm{~h}$ after Li-SWT treatment with a duration of $18 \mathrm{~h}$ revealed no change in the ability of human Li-SWT myoblasts to migrate compared to controls. (TIFF $1198 \mathrm{~kb}$ )

Additional file 3: $\mathrm{HE}$ stainings of the regeneration process after CTX injury with and without Li-SWT on paired hindlimbs. The extend of injury caused by cardiotoxin varied between hindlimbs, but overall no adverse affects of Li-SWT was found. White arrows point to normal muscle areas, while black arrows point to regenerative areas. In the upper right corner of each picture the regenerative area is upscaled. Scalebars represents $100 \mu \mathrm{m}$. (TIFF $8648 \mathrm{~kb}$ )

\section{Abbreviations}

Li-SWT: Low intensity shockwave therapy; SC: Satellite cells, BrdU: Bromodeoxyuridine

\section{Acknowledgements}

We would like to thank Lone Christiansen for technical support.

\section{Funding}

The study was financially supported by The Lundbeck Foundation, a collaborative Indo-Danish grant from the Government of India, Department of Biotechnology (BT/IN/Denmark/02/PDN/2011 DTD 26-05-11) and the Danish Council for Strategic Research (10-093757) and grants from Odense University Hospital.

\section{Availability of data and materials}

All datasets on which the conclusions of the manuscript rely are presented in the main paper and additional supporting files.

\section{Authors contributions}

LKH: Study design, Collection and assembly of data, data analysis and interpretation, manuscript writing. HDS: Conception and design, data interpretation, manuscript writing. LL: Study design, provision of Duolith SD1 machine. KR: planned and carried out the experiment on the effect of shock wave on cell viability, carried out these experiments VM: planned the effect of shock wave on cell proliferation and viability JS: Conception and design, financial support, collection and data assembly, data analysis and interpretation, manuscript writing. All authors: reviewed and approved the manuscript.

\section{Ethics approval and consent to participate}

Human muscle tissue donors included in this study gave written informed consent and The Regional Scientific Ethical Committees for Southern Denmark approved the study (S-20070079).

The animals were treated in accordance to the Danish law on animal experiments and European Directive 2012/63. The study was approved by the Danish Animal Experiments Inspectorate (2013-15-2934-00844).

\section{Consent for publication}

Not applicable.

\section{Competing interest}

The authors declare that they have no competing interests.

\section{Publisher's Note}

Springer Nature remains neutral with regard to jurisdictional claims in published maps and institutional affiliations.

\section{Author details}

'Department of Clinical Pathology, SDU Muscle Research Cluster (SMRC), Odense University Hospital, Odense, Denmark. Institute of Clinical Research, Faculty of Health Science, University of Southern Denmark, Odense, Denmark. ${ }^{3}$ Department of Urology, Odense University Hospital, Odense, Denmark. ${ }^{4}$ Paediatric Orthopaedic Unit and Center for Stem Cell Research, Christian Medical Centre, Vellore, India.

Received: 30 November 2016 Accepted: 28 November 2017

Published online: 29 December 2017

\section{References}

1. Skuk D, Tremblay JP. Intramuscular cell transplantation as a potential treatment of myopathies: clinical and preclinical relevant data. Expert Opin Biol Ther. 2011;11:359-74.

2. Fan $\mathrm{Y}$, Maley $\mathrm{M}$, Beilharz $\mathrm{M}$, et al. Rapid death of injected myoblasts in myoblast transfer therapy. Muscle Nerve. 1996;19:853-60.

3. Smythe GM, Hodgetts SI, Grounds MD. Immunobiology and the future of myoblast transfer therapy. Molecular therapy : the journal of the American Society of Gene Therapy. 2000;1:304-13.

4. Boldrin L, Elvassore N, Malerba A, et al. Satellite cells delivered by micropatterned scaffolds: a new strategy for cell transplantation in muscle diseases. Tissue Eng. 2007;13:253-62.

5. Wolf MT, Dearth CL, Sonnenberg SB, et al. Naturally derived and synthetic scaffolds for skeletal muscle reconstruction. Adv Drug Deliv Rev. 2014;

6. Bramfeldt H, Sabra G, Centis V, et al. Scaffold vascularization: a challenge for three-dimensional tissue engineering. Curr Med Chem. 2010;17:3944-67.

7. Suzuki K, Murtuza B, Smolenski RT, et al. Cell transplantation for the treatment of acute myocardial infarction using vascular endothelial growth factor-expressing skeletal myoblasts. Circulation. 2001;104:1207-12.

8. Borselli C, Cezar CA, Shvartsman D, et al. The role of multifunctional delivery scaffold in the ability of cultured myoblasts to promote muscle regeneration. Biomaterials. 2011;32:8905-14.

9. Wang CJ. Extracorporeal shockwave therapy in musculoskeletal disorders. J Orthop Surg Res. 2012;7:11.

10. Wang $\mathrm{CJ}$, Huang $\mathrm{HY}$, Pai $\mathrm{CH}$. Shock wave-enhanced neovascularization at the tendon-bone junction: an experiment in dogs. The Journal of foot and 
ankle surgery : official publication of the American College of Foot and Ankle Surgeons. 2002;41:16-22.

11. Hausner T, Pajer K, Halat $G$, et al. Improved rate of peripheral nerve regeneration induced by extracorporeal shock wave treatment in the rat. Exp Neurol. 2012;236:363-70.

12. Ogden JA, Toth-Kischkat A, Schultheiss R. Principles of shock wave therapy. Clin Orthop Relat Res. 2001:8-17.

13. van der Worp $\mathrm{H}$, van den Akker-Scheek I, van Schie H, et al. ESWT for tendinopathy: technology and clinical implications. Knee surgery, sports traumatology, arthroscopy : official journal of the ESSKA. 2013;21:1451-8.

14. Ohl CD, Wolfrum B. Detachment and sonoporation of adherent HeLa-cells by shock wave-induced cavitation. Biochim Biophys Acta. 2003;1624:131-8.

15. Khan KM, Scott A. Mechanotherapy: how physical therapists' prescription of exercise promotes tissue repair. Br J Sports Med. 2009;43:247-52.

16. Vetrano M, d'Alessandro F, Torrisi MR, et al. Extracorporeal shock wave therapy promotes cell proliferation and collagen synthesis of primary cultured human tenocytes. Knee surgery, sports traumatology, arthroscopy : official journal of the ESSKA. 2011;19:2159-68.

17. Leone L, Vetrano M, Ranieri D, et al. Extracorporeal shock wave treatment (ESWT) improves in vitro functional activities of ruptured human tendonderived tenocytes. PLoS One. 2012;7:e49759.

18. Hofmann A, Ritz U, Hessmann MH, et al. Extracorporeal shock wavemediated changes in proliferation, differentiation, and gene expression of human osteoblasts. J Trauma. 2008;65:1402-10.

19. Zhang $X$, Yan X, Wang C, et al. The dose-effect relationship in extracorporeal shock wave therapy: the optimal parameter for extracorporeal shock wave therapy. J Surg Res. 2014;186:484-92.

20. Nurzynska D, Di Meglio F, Castaldo C, et al. Shock waves activate in vitro cultured progenitors and precursors of cardiac cell lineages from the human heart. Ultrasound Med Biol. 2008;34:334-42.

21. Yan $X$, Zeng B, Chai Y, et al. Improvement of blood flow, expression of nitric oxide, and vascular endothelial growth factor by low-energy shockwave therapy in random-pattern skin flap model. Ann Plast Surg. 2008;61:646-53.

22. Mittermayr R, Hartinger J, Antonic V, et al. Extracorporeal shock wave therapy (ESWT) minimizes ischemic tissue necrosis irrespective of application time and promotes tissue revascularization by stimulating angiogenesis. Ann Surg. 2011;253:1024-32.

23. Stojadinovic A, Elster EA, Anam K, et al. Angiogenic response to extracorporeal shock wave treatment in murine skin isografts. Angiogenesis. 2008:11:369-80.

24. Tepekoylu C, Wang FS, Kozaryn R, et al. Shock wave treatment induces angiogenesis and mobilizes endogenous CD31/CD34-positive endothelial cells in a hindlimb ischemia model: implications for angiogenesis and vasculogenesis. J Thorac Cardiovasc Surg. 2013;146:971-8.

25. Krokowicz L, Mielniczuk M, Drews M, et al. Long-term follow up of the effects of extracorporeal shockwave therapy (ESWT) on microcirculation in a denervated muscle flap. Polski przeglad chirurgiczny. 2011;83:325-33.

26. Krokowicz L, Cwykiel J, Klimczak A, et al. Pulsed acoustic cellular treatment induces expression of proangiogenic factors and chemokines in muscle flaps. J Trauma. 2010;69:1448-56.

27. ISMST - International Society for Medical Shockwave Treatment. Shockwave Indications. Available at https://www.shockwavetherapy.org/about-eswt/ indications/. Accessed 4 Feb 2015.

28. Kraus $M$, Reinhart $E$, Krause $H$, et al. Low energy extracorporeal shockwave therapy (ESWT) for treatment of myogelosis of the masseter muscle. MundKiefer- und Gesichtschirurgie : MKG. 1999;3:20-3.

29. Gonkova MI, llieva EM, Ferriero G, et al. Effect of radial shock wave therapy on muscle spasticity in children with cerebral palsy. International journal of rehabilitation research Internationale Zeitschrift fur Rehabilitationsforschung Revue internationale de recherches de readaptation. 2013;36:284-90.

30. Ciccone MM, Notarnicola A, Scicchitano P, et al. Shockwave therapy in patients with peripheral artery disease. Adv Ther. 2012;29:698-707.

31. Sellathurai J, Cheedipudi S, Dhawan J, et al. A novel in vitro model for studying quiescence and activation of primary isolated human myoblasts. PLoS One. 2013;8:e64067

32. Liu N, Nelson BR, Bezprozvannaya S, et al. Requirement of MEF2A, C, and D for skeletal muscle regeneration. Proc Natl Acad Sci U S A. 2014;11:4109-14.

33. Fulda S, Gorman AM, Hori O, et al. Cellular stress responses: cell survival and cell death. International journal of cell biology. 2010;2010:214074.

34. Karalaki M, Fili S, Philippou A, et al. Muscle regeneration: cellular and molecular events. In vivo (Athens, Greece). 2009;23:779-96.
35. Lefaucheur JP, Sebille A. The cellular events of injured muscle regeneration depend on the nature of the injury. Neuromuscular disorders : NMD. 1995;5:501-9.

36. Wang CJ, Yang YJ, Huang CC. The effects of shockwave on systemic concentrations of nitric oxide level, angiogenesis and osteogenesis factors in hip necrosis. Rheumatol Int. 2011;31:871-7.

37. Seghezzi G, Patel S, Ren CJ, et al. Fibroblast growth factor-2 (FGF-2) induces vascular endothelial growth factor (VEGF) expression in the endothelial cells of forming capillaries: an autocrine mechanism contributing to angiogenesis. J Cell Biol. 1998;141:1659-73.

38. Qiu $X$, Lin $G$, Xin Z, et al. Effects of low-energy shockwave therapy on the erectile function and tissue of a diabetic rat model. J Sex Med. 2013;10:738-46.

39. Wang CJ, Yang KD, Ko JY, et al. The effects of shockwave on bone healing and systemic concentrations of nitric oxide (NO), TGF-beta1, VEGF and BMP2 in long bone non-unions. Nitric oxide : biology and chemistry / official journal of the Nitric Oxide Society. 2009;20:298-303.

40. Hausdorf J, Sievers B, Schmitt-Sody M, et al. Stimulation of bone growth factor synthesis in human osteoblasts and fibroblasts after extracorporeal shock wave application. Arch Orthop Trauma Surg. 2011;131:303-9.

41. Chandran R, Knobloch TJ, Anghelina M, et al. Biomechanical signals upregulate myogenic gene induction in the presence or absence of inflammation. American journal of physiology Cell physiology. 2007;293:C267-76.

42. White $C R$, Frangos JA. The shear stress of it all: the cell membrane and mechanochemical transduction. Philos Trans R Soc Lond Ser B Biol Sci. 2007;362:1459-67.

43. Holfeld J, Tepekoylu C, Kozaryn R, et al. Shockwave therapy differentially stimulates endothelial cells: implications on the control of inflammation via toll-like receptor 3. Inflammation. 2014;37:65-70.

44. Modulevsky DJ, Tremblay D, Gullekson C, et al. The physical interaction of myoblasts with the microenvironment during remodeling of the cytoarchitecture. PLoS One. 2012;7:e45329.

45. Moosavi-Nejad SF, Hosseini SH, Satoh M, et al. Shock wave induced cytoskeletal and morphological deformations in a human renal carcinoma cell line. Cancer Sci. 2006;97:296-304.

46. $\mathrm{Xu} \mathrm{JK}$, Chen $\mathrm{HJ}$, Li XD, et al. Optimal intensity shock wave promotes the adhesion and migration of rat osteoblasts via integrin beta1-mediated expression of phosphorylated focal adhesion kinase. J Biol Chem. 2012;287: 26200-12.

47. Kuo YR, Wang CT, Wang FS, et al. Extracorporeal shock wave treatment modulates skin fibroblast recruitment and leukocyte infiltration for enhancing extended skin-flap survival. Wound repair and regeneration : official publication of the Wound Healing Society [and] the European Tissue Repair Society. 2009;17:80-7.

48. Smith C, Kruger MJ, Smith RM et al. The inflammatory response to skeletal muscle injury: illuminating complexities. Sports medicine (Auckland, NZ) 2008;38:947-969.

49. Silvestre JS, Smadja DM, Levy BI. Postischemic revascularization: from cellular and molecular mechanisms to clinical applications. Physiol Rev. 2013;93: 1743-802.

50. Davis TA, Stojadinovic A, Anam K, et al. Extracorporeal shock wave therapy suppresses the early proinflammatory immune response to a severe cutaneous burn injury. Int Wound J. 2009;6:11-21.

51. Juhas M, Bursac N. Engineering skeletal muscle repair. Curr Opin Biotechnol. 2013;24:880-6

52. Wozniak AC, Anderson JE. Nitric oxide-dependence of satellite stem cell activation and quiescence on normal skeletal muscle fibers. Developmental dynamics : an official publication of the American Association of Anatomists. 2007:236:240-50

53. Juffer P, Bakker AD, Klein-Nulend J, et al. Mechanical loading by fluid shear stress of myotube glycocalyx stimulates growth factor expression and nitric oxide production. Cell Biochem Biophys. 2014;69:411-9.

54. Lowry JL, Brovkovych V, Zhang Y, et al. Endothelial nitric-oxide synthase activation generates an inducible nitric-oxide synthase-like output of nitric oxide in inflamed endothelium. J Biol Chem. 2013;288:4174-93.

55. Mariotto S, de Prati AC, Cavalieri E, et al. Extracorporeal shock wave therapy in inflammatory diseases: molecular mechanism that triggers antiinflammatory action. Curr Med Chem. 2009;16:2366-72.

56. Davis ME, Grumbach IM, Fukai T, et al. Shear stress regulates endothelial nitric-oxide synthase promoter activity through nuclear factor kappaB binding. J Biol Chem. 2004;279:163-8.

57. Fagiani $\mathrm{E}$, Christofori G. Angiopoietins in angiogenesis. Cancer Lett. 2013; 328:18-26. 